\title{
1 Title: iDEP-assisted isolation of insulin secretory vesicles
}

\section{Authors}

Mahta Barekatain ${ }^{1 *}$, Yameng Liu² ${ }^{2}$, Zhongying Wang 3 , Vadim Cherezov ${ }^{1}$, Scott E. Fraser ${ }^{3}$, Kate L White ${ }^{1}$,

4 and Mark A. Hayes ${ }^{2}$

\section{$5 \quad$ Affiliations}

${ }^{1}$ Department of Chemistry, Bridge Institute, USC Michelson Center for Convergent Bioscience, University of Southern California, Los Angeles, CA 90089, USA.

${ }^{2}$ School of Molecular Sciences, Arizona State University, Tempe, AZ 85287, USA

${ }^{3}$ Department of Biological Sciences, Bridge Institute, USC Michelson Center for Convergent Bioscience, University of Southern California, Los Angeles, CA 90089, USA.

* Denotes equal contribution.

Organelle heterogeneity and inter-organelle associations within a single cell contribute to the limited sensitivity of current organelle separation techniques, thus hindering organelle subpopulation characterization. Here we use direct current insulator-based dielectrophoresis (DC-iDEP) as an unbiased separation method and demonstrate

17 its capability by identifying distinct distribution patterns of insulin vesicles from pancreatic $\beta$-cells. A multiple voltage DC-iDEP strategy with increased range and sensitivity has been applied, and a differentiation factor (ratio of

19 electrokinetic to dielectrophoretic mobility) has been used to characterize features of insulin vesicle distribution

20 patterns. We observed a significant difference in the distribution pattern of insulin vesicles isolated from glucose-

21 stimulated cells relative to unstimulated cells, in accordance with functional maturation of vesicles upon glucose

22 stimulation, and interpret this to be indicative of high-resolution separation of vesicle subpopulation. DC-iDEP

23 provides a path for future characterization of subtle biochemical differences of organelle subpopulations within any

24 biological system.

\section{INTRODUCTION}

Functional cell-to-cell heterogeneity within a given cell type arises from differences in genomic, epigenomic,

28 transcriptomic, and proteomic ${ }^{1}$ components and their specific subcellular localizations. Evidence for subpopulations 
29 of organelles has been obtained for synaptic vesicles ${ }^{2}$, mitochondria $^{3}$, and insulin secretory vesicles ${ }^{4}$, among others.

30 The different subpopulations of organelles may represent differences in age, maturation, or even function because of

31 variations in biochemical composition. To better characterize cell function, we must identify the number of organelle

32 subpopulations that exist and their distinct functional states. Understanding the dynamics of organelle composition

33 and identifying functional subpopulations is a fundamental aspect of cell biology that is limited by lack of appropriate

34 experimental methods. To address this gap in technology, we developed a new approach for organelle isolation.

35 Ultimately, this approach will be useful for follow up biochemical analyses such as proteomics, functional assays, and

36 imaging.

37 Conventional separation methods have proved powerful for numerous biological analyses. This process

38 traditionally consists of iterative centrifugation steps ${ }^{5}$ to isolate populations of targeted organelles for follow up

39 analysis with mass spectrometric or other omics-scale assays ${ }^{6}$. Gradient columns have successfully isolated clathrin-

40 coated vesicles $^{7}$, lipid droplets ${ }^{8}$, mitochondria, and endoplasmic reticulum (ER) populations ${ }^{9}$, from other organelles,

41 based on differences in organelle densities. However, subpopulations of a given organelle with similar buoyant

42 densities are often hard to differentiate and separate in these columns. Immunoisolation of organelles, although

43 yielding pure populations, is limited by the need for specific organelle markers ${ }^{10}$. Electrophoretic approaches, such as

44 Free-Flow Electrophoresis (FFE), have been adopted for use in micro-fluidic separation of organelles ${ }^{11}$, enabling

45 downstream analyses of the enriched fractions in the absence of major contaminants; however, such electrophoretic

46 separations rely on charge differences of the components. Although powerful, each of these traditional isolation

47 approaches lack sufficient sensitivity and robustness to isolate subpopulations of an organelle, except for smooth

48 versus rough $\mathrm{ER}^{12}$, for biochemical characterizations such as proteomic analysis or imaging. Thus, an alternative

49 approach to organelle isolation will be beneficial to define organelle subpopulations more accurately.

50 Direct current insulator-based dielectrophoresis (DC-iDEP) provides a valuable tool for separating

51 subpopulations of bioparticles with high resolution including viruses, bacteria, organelles, and proteins ${ }^{13-16}$. This

52 approach offers a wide dynamic range, as it has been used for separations ranging from neural progenitors and stem

53 cells ${ }^{17}$ to resistant versus susceptible strains of cellular pathogens ${ }^{15,18}$. In DC-iDEP, subtle biophysical differences can

54 be distinguished by the differences in dielectrophoretic (DEP) and electrokinetic (EK) forces that result from a rich

55 set of distinguishing factors associated with the radius, zeta potential, permittivity, interfacial polarizability and

56 conductivity of the particles, to name but a few ${ }^{18-22}$. DC-iDEP is well-suited for assaying subpopulations of organelles, 
57 where differentiating factors are not known, because it is unbiased compared to approaches that rely on defined 58 biomarkers. DC-iDEP can be used as a discovery-based approach to interrogate a broader spectrum of organelle 59 subpopulations ${ }^{17}$ because the bioparticle separations occur quickly and the required sample volume is small ${ }^{14,23,24}$. 60 Moreover, DC-iDEP is extremely sensitive to subtle biophysical differences of bioparticle or organelle identities and 61 has been shown to be reproducible ${ }^{15,17}$. Here, we demonstrate the power of DC-iDEP in organelle isolation, by using 62 it to investigate subtle differences in the subpopulations of insulin vesicles in pancreatic $\beta$-cells. Pancreatic $\beta$-cells are responsible for secreting insulin in a tightly regulated process that is key to maintaining

64 glucose homeostasis. Insulin vesicles undergo a complex functional maturation process that is required for proper 65 secretion of insulin and this process is dysregulated in disease state such as diabetes ${ }^{4}$. Immature insulin vesicles act as 66 a sorting compartment ${ }^{25,26}$ and mature into two distinct pools of functional vesicles within the cell: the readily 67 releasable pool and the reserve pool ${ }^{27-30}$ (Fig. 1A). This apparent heterogeneity among subpopulations of insulin 68 vesicles is thought to arise in part from the maturation process of insulin vesicles ${ }^{31,32}$ as well as their age, mobility, 69 localization and modifications of insulin vesicle membrane proteins within the cell ${ }^{4,33-35}$. However, the associated 70 biochemical constituents of insulin vesicles have remained elusive in the absence of sensitive isolation approaches.

71 The importance of insulin vesicles in glucose homeostasis has led several groups to attempt to isolate and characterize

72 the insulin vesicles ${ }^{36,37}$. While these studies and follow-up proteomics analysis of the isolated vesicles ${ }^{39-42}$ have

73 provided insights into the biochemistry of these vesicles, there's very little overlap in protein IDs associated with these

74 organelles from different studies (Fig. 1B). Thus, there is a need for more robust isolation methods that can 75 reproducibly differentiate between the heterogeneous subpopulations of insulin vesicles, among other organelles, and 76 allow for their downstream characterization. In this study, a new scanning voltage DC-iDEP separation strategy has been applied to immunolabeled insulin

78 vesicles of the INS-1E $\beta$-cells (rat insulinoma cell line ${ }^{43}$ ) and has been shown to separate the full range of insulin 79 vesicle subpopulations with improved resolution within multiple ranges of biophysical parameters (Fig. 1C). We 80 demonstrate that glucose treatment, which has been shown to influence the dynamics of this organelle within the $\beta$ 81 cell $^{44}$, affects the biophysical characteristics associated with the vesicle subpopulations captured within our DC-iDEP 82 device. This method allows discovery of subpopulations with distinct biophysical properties amongst insulin vesicles 83 from untreated cells ( $\mathrm{n}$-insulin vesicles) and $25 \mathrm{mM}$ glucose-treated cells (g-insulin vesicles). Our observations are 84 consistent with previous studies where a pronounced shift in the molecular density of the insulin vesicles was noted 
85 under the two conditions ${ }^{44}$. This study substantiates the sensitivity of DC-iDEP separation technique in resolving

86 subpopulations of insulin vesicles, among other organelles, and opens the avenue for numerous studies of the

87 biochemical constituents where complex and heterogeneous populations of organelles are of interest.

\section{METHODS}

90 No statistical methods were used to predetermine sample size. The experiments were not randomized, and

91 investigators were not blinded to allocation during experiments and outcome assessment.

\section{Cell culture}

INS-1E cells (Addex Bio C0018009; RRID (Research Resource Identifiers) accession number: CVCL_0351)

were cultured according to the supplier's protocol. Briefly, cells were seeded in RPMI 1640 media (modified to contain $2 \mathrm{mM}$ L-glutamine; $10 \mathrm{mM}$ HEPES pH 7.2, $1 \mathrm{mM}$ sodium pyruvate, $2 \mathrm{~g} / \mathrm{L}$ glucose, and $1.5 \mathrm{~g} / \mathrm{L}$ sodium bicarbonate, $50 \mu \mathrm{M}$ 2-mercaptoethanol, $100 \mathrm{U} / \mathrm{mL}$ penicillin, and $100 \mu \mathrm{g} / \mathrm{mL}$ streptomycin; sterile filtered through $0.22 \mu \mathrm{m}$ filter) supplemented with $10 \%$ fetal bovine serum (FBS) and grown to $80 \%$ confluency. Cells were plated at a density of $10^{5}$ cells $/ \mathrm{cm}^{2}$ in a 24 well plate for the glucose sensitivity assay and incubated at $37^{\circ} \mathrm{C}$ with $5 \% \mathrm{CO}_{2}$ in growth media for 4-5 days. Cells were pretreated at 60-80\% confluency with Krebs-Ringer bicarbonate HEPES (KRBH) buffer (135 $\mathrm{mM} \mathrm{NaCl}, 3.6 \mathrm{mM} \mathrm{KCl}, 5 \mathrm{mM} \mathrm{NaHCO}_{3}, 0.5 \mathrm{mM} \mathrm{NaH}_{2} \mathrm{PO}_{4}, 0.5 \mathrm{mM} \mathrm{MgCl}_{2}, 1.5 \mathrm{mM} \mathrm{CaCl}_{2}, 10 \mathrm{mM} \mathrm{HEPES}, \mathrm{pH} 7.4$, and $0.1 \%$ Bovine Serum Albumin (BSA); made fresh within 7 days of use) free of glucose and incubated at $37^{\circ} \mathrm{C}$ with

$1035 \% \mathrm{CO}_{2}$ for $30 \mathrm{~min}$. They were then stimulated for $30 \mathrm{~min}$ at $37^{\circ} \mathrm{C}$ using $\mathrm{KRBH}$ buffers containing 1.1, 5.6, 8.4, 11.1, 104 16.7, and $25 \mathrm{mM}$ glucose, in the presence of house-made protease inhibitor (PI) cocktail (0.5 M AEBSF, $1 \mathrm{mM} \mathrm{E-64,}$ $1.13 \mathrm{mM}$ leupeptin, and $151.36 \mu \mathrm{M}$ aprotinin). KRBH buffer was removed and saved from cells for downstream

106 analysis. Insulin secretion in response to increasing concentrations of glucose was confirmed in an Enzyme Linked 107 Immuno-Sorbent Assay (ELISA) (Mercodia 10-1250-01) following the manufacturer's manual (Fig. S1). For each 108 biological replica, cells were plated at a density of $4 \times 10^{4}$ cells $/ \mathrm{cm}^{2}$ in a 5-layer cell chamber (VWR 76045-402) to 109 yield enough material for completing the experiment. All cell stacks were at least $95 \%$ viable after the harvest with $1100.05 \%$ trypsin. For glucose treatment, near $80 \%$ confluent cells were gently rinsed with dialyzed phosphate-buffered 111 saline (dPBS) twice and starved in a KRBH buffer with no glucose for $30 \mathrm{~min}$, followed by a 30 min stimulation of 112 insulin release by KRBH buffer supplemented with $25 \mathrm{mM}$ glucose. Cells were then harvested by mild trypsinization. 


\section{Colocalization of synaptotagmin $\mathrm{V}$ and insulin vesicles}

Cells were grown on Nunc Lab-Tek II Chamber Slide, precoated with poly-L-lysine. Cells were fixed with 4\% ice-cold PFA for $10 \mathrm{~min}$, and then stained with antibody cocktail (Rabbit anti-insulin antibody (Abcam ab181547), 1:1000; Mouse anti-synaptotagmin V (BD Biosciences, 612284), 1:1000) in 0.5\% BSA, $0.2 \%$ saponin, $1 \%$ FBS of PBS buffer for $1 \mathrm{~h}$ at room temperature (RT). After three washes with PBST for $10 \mathrm{~min}$, cells were incubated for 40 min with secondary antibody cocktail (Goat anti-rabbit IgG H\&L, Alexa 488 (Abcam ab150077) 1:1000; Goat antiMouse IgG (H+L) Cross-Adsorbed Secondary Antibody, Alexa Fluor 647 (Invitrogen A-21235), 1:1000). Cells were then mounted in ProLong ${ }^{\mathrm{TM}}$ Glass Antifade Mountant with NucBlue ${ }^{\mathrm{TM}}$ Stain (Thermo Fisher Scientific P36981) and cured for $24 \mathrm{~h}$ before imaging.

\section{Microscopy imaging}

A Leica SP8 FALCON with DIVE laser scanning microscope was used for confocal imaging. Image acquisition was performed using 63x/1.4NA oil immersion objective, using $1024 x 1024$ format at $400 \mathrm{~Hz}$, with 0.03 $\mu \mathrm{m}$ pixel size, and pinhole set to $1 \mathrm{AU}$. The signal was collected with $405 \mathrm{~nm}, 499 \mathrm{~nm}$, and $653 \mathrm{~nm}$ excitations and 410-494 nm, 504-621 nm, 658-776 nm emissions for nuclei, insulin, and synaptotagmin V by HyD detector. The images were deconvoluted by LAS X, lightening module. The refraction index was set to 1.52 for processing.

\section{Insulin secretory vesicle enrichment}

Following trypsinization, all the steps were performed at $4^{\circ} \mathrm{C}$. Cells were gently washed twice with PBS, mM MES, 1 mM EGTA, 1 mM MgSO 4 , pH 6.3) supplemented with house-made PI cocktail (0.5 M AEBSF, $1 \mathrm{mM}$ E-64, $1.13 \mathrm{mM}$ leupeptin, and $151.36 \mu \mathrm{M}$ aprotinin). Cell debris was collected by centrifugation at $600 \mathrm{x}$ for $10 \mathrm{mins}$ and re-homogenized as described above to lyse the remaining intact cells, followed by a second spin at 600x g. Supernatants were pooled and centrifuged at 5,400x g for $15 \mathrm{~min}$ to remove mitochondria, ER, and other subcellular compartments of similar density. The pellet was discarded, and the supernatant was centrifuged at 35,000x g for 30 min to sediment insulin vesicles, among other contaminants, yielding up to $\sim 5 \mu \mathrm{g}$ of dry material per every million cells. This pellet was resuspended in $\sim 450 \mu \mathrm{L} \mathrm{HB}$ and loaded on a density gradient column formed by layering decreasing densities of OptiPrep density media (Sigma-Aldrich D1556) and $\mathrm{HB}$ in $0.9 \mathrm{~mL}$ fractions of 40, 35, 30, 25, 
141 and 20 percent OptiPrep in an open-top thin-wall polypropylene tube (Beckman 326819). The density column was

142 then spun in a SW55i Beckman rotor of an ultracentrifuge at 160,000x $\mathrm{g}$ for $8 \mathrm{~h}$ to fractionate the insulin vesicle-

143 containing population. Insulin vesicle subpopulations were isolated in fractions of $400 \mu \mathrm{L}$, and ELISA and western

144 blotting (WB) were used to identify the fractions most enriched in insulin. A similar dilution was applied to all the

145 fractions. The manufacturer's manual was followed for ELISA (Mercodia 10-1250-01), (Fig. S2A). For WB, fraction

146 samples were mixed with 4X NuPAGETM LDS sample buffer (Invitrogen NP0007), loaded on a 15-well NuPAGETM

1474 to $12 \%$ bis-tris gel (Invitrogen NP0323PK2) and run in a mini-gel tank (Life Technologies A25977) at 200V for 30

148 min (Bio-Rad 1645050). Protein was then transferred to a PVDF membrane using iBlot 2 Transfer Stacks

149 (Invitrogen IB24002) in iBlot 2 dry blotting device (Invitrogen IB21001). The membrane was blocked using 5\% BSA,

150 then cut according to marker protein size and incubated with antibodies against marker proteins for mitochondria

151 (Cytochrome c Antibody; Novus Biologicals NB100-56503), ER (SEC61B Polyclonal Antibody; Life Technologies

152 PA3015), and insulin vesicles (synaptotagmin V; Thermo Fisher Scientific PA5-44987; RRID: AB_2610517) at RT

153 for 2-5 h. Membranes were then washed with 0.1\% Tween supplemented PBS (PBST) twice and incubated with the

154 secondary antibody ((anti-rabbit IgG), (anti-mouse IgG)) at RT for $1 \mathrm{~h}$. Membranes were washed with PBST 2-3 times

155 and bands were visualized upon addition of SigmaFast BCIP ${ }^{\circledR} /$ NBT tablets (Sigma B5655) (Fig. S2B). Fractions

156 containing the highest insulin levels and high concentration of the vesicle marker synaptotagmin V were regarded as

157 insulin vesicle samples and were further tested for dynamic light scattering (DLS) using Wyatt Technology's Mobius

158 to confirm the size distribution of particles corresponding to the insulin vesicle diameter, reportedly $200-500 \mathrm{~nm}$. Data

159 was analyzed in DYNAMICS and manually corrected against the control (HB) (Fig. S2C). Insulin vesicle fractions

160 confirmed to have the expected insulin vesicle diameter by DLS were then pooled together and spun down at 35,000x

$161 \mathrm{~g}$ for 15 min to sediment the insulin vesicles.

\section{Immunolabeling of insulin vesicles}

The pellet was resuspended in HB and incubated with anti-synaptotagmin V (Thermo Fisher Scientific PA5-

44987; RRID: AB_2610517) to tag an insulin vesicle marker, which served as a primary antibody that was further 


\section{Device fabrication}

The design and fabrication methods of the separation device were described in prior publications ${ }^{45}$. The

171 device contains a 27-gate sawtooth channel with a depth of approximately $20 \mu \mathrm{m}$ and a length of $3.5 \mathrm{~cm}$ from inlet to

172 outlet (Fig. 2C). The distance between two paired triangle tips (gates) decreases from $73 \mu \mathrm{m}$ to $25 \mu \mathrm{m}$ in the channel.

173 The gate size decreases approximately $5 \mu \mathrm{m}$ after every 3 repeats. Direct current was applied to the device between

174 the inlet and outlet. The potentials were between $0-1800 \mathrm{~V}$ for testing.

175 The microfluidic devices were fabricated by standard soft lithographic technique as described previously ${ }^{45}$.

176 The design of the channel was created by AutoCAD (Autodesk, Inc., San Rafael, CA) and was used for fabricating a

177 photomask. The channel was created by exposing AZ P4620 positive photoresist (AZ Electronic Materials,

178 Branchburg, NJ) on Si wafer CEM388SS (Shin-Etsu MicroSi, Inc., Phoenix, AZ) by contact lithography. Extra

179 materials were removed from the Si wafer. A weight of $22 \mathrm{~g}$ of polydimethylsiloxane (PDMS, Sylgard 184,

180 Dow/Corning, Midland, MI) was used to fabricate four channels simultaneously. The PDMS mixture was placed on

181 the Si wafer template and left to stand for $30 \mathrm{~min}$ to allow bubbles to dissipate, and then it was baked for $1 \mathrm{~h}$ at a

182 temperature of $80^{\circ} \mathrm{C}$. Holes $2.5 \mathrm{~mm}$ in diameter were punched for inlet and outlet reservoirs. Each channel was capped

183 with a glass microscope slide to fabricate the enclosed channels after cleaning and activation by plasma cleaner

184 (Harrick Plasma, Ithaca, NY, USA) with a voltage of $50 \mathrm{kV}$.

\section{Electric field simulations}

Finite element modeling (COMSOL, Inc., Burlington, MA) of the distribution of the electric field in the

188 microchannel was performed as previously detailed ${ }^{45}$. The AC/DC module was used to interrogate the $\vec{E}, \nabla|\vec{E}|^{2}$, and

$189 \frac{\nabla|\vec{E}|^{2}}{E^{2}} \cdot \vec{E}$ in an accurately scaled 2D model of the microchannel.

\section{DC-iDEP}

192 The separation channel was treated with 5\% (w/v) BSA for 15 min followed by a wash with LCHB. A volume of 15

$193 \mu \mathrm{L}$ of the insulin vesicle sample was introduced to the device from the inlet. This sample fraction contains particles

$194 \sim 75 \%$ of which have radii characteristic of insulin vesicle as apparent from DLS experiments (Fig. 2SC). The volume

195 in each reservoir was maintained with LCHB to prevent pressure induced flow. Direct current was applied at 600V, 
$900 \mathrm{~V}, 1200 \mathrm{~V}, 1500 \mathrm{~V}$, and $1800 \mathrm{~V}$ between the inlet and outlet and particles were driven through the channel by the

EK force experienced.

\section{Imaging}

199 Images and recordings were acquired using an Olympus IX70 inverted microscope with 4X, N.A. 0.16, and 20X, N.A.

200 0.40, objectives. The 20X objective was used to inspect the channel to confirm that the device was properly formed.

201 The 4X objective was used in recording the intensity of the insulin vesicle signal shown in Figure 1. A mercury short 202 arc lamp (H30102 w/2, OSRAM) and a triple band pass cube (Olympus, Center Valley, PA) were used for sample

203 illumination and detection (Excitation:400/15-495/15-570/25; Dichroic:410-510-590; Emission:460/20-530/30-

204 625/50). Fluorescent intensities of immunolabeled insulin vesicles were recorded using the $4 \mathrm{X}$ objective by a 205 LightWise Allegro camera (LW-AL-CMV12000, USB3, 0059-0737-B, Imaging Solutions Group) after the voltage 206 had been applied $90 \mathrm{~s}$. Images were recorded from 3-4 biological replicates at each gate (27 total gates) for any given 207 voltage. Images were further processed in ImageJ (NIH, freeware). The intensity at each gate was recorded along with 208 the intensity of a nearby open area of the channel (image intensity background), which was subtracted from the 209 intensity at each gate to adjust for any variation in illumination intensity. The data for each applied voltage value was 210 normalized to the highest intensity within that data set.

\section{Theory}

The forces exerted on bioparticles in the microfluidic device in the presence of direct current include

214 dielectrophoretic (DEP) and electrokinetic (EK) forces. Separation of subpopulations is achieved based on the 215 different magnitude of the forces each bioparticle experiences, related to the properties of the particles, including their 216 radius, conductivity, and zeta potential.

217 The EK mobility, $\mu_{E K}$, and velocity, $\vec{v}_{E K}$, are described as: mobility, $\mu_{D E P}$ and velocity, $\vec{v}_{D E P}$ can be expressed as:

$$
\begin{gathered}
\mu_{E K}=\mu_{E P}+\mu_{E O F} \\
\vec{v}_{E K}=\mu_{E K} \vec{E}
\end{gathered}
$$

$$
\mu_{D E P}=\frac{\varepsilon_{m} r^{2} f_{C M}}{3 \eta}
$$




$$
\vec{v}_{D E P}=\mu_{D E P} \nabla|\vec{E}|^{2}
$$

224 where $r$ is the radius of the particle, $f_{C M}$ is the Clausius-Mossotti factor, $\varepsilon_{m}$ is the dielectric constant of the solution,

225 and $\eta$ is the viscosity.

226 A combination of biophysical properties of the particles, such as insulin vesicles, determines the location 227 where they will be captured in a microfluidic device. Capture occurs when the EK velocity of the particle is equal to 228 that of DEP. The condition is: where $\vec{E}$ is the electric field intensity, $\vec{J}$ is the particle flux, and $\nabla|\vec{E}|$ is the gradient of the electric field. The ratio of EK to DEP mobilities (EKMr, $\frac{\mu_{E K}}{\mu_{D E P}}$ ) can be used to characterize the biophysical properties of different

233 subpopulations ${ }^{17}$. The EKMr of an insulin vesicle is larger than $\frac{\nabla|\vec{E}|^{2}}{E^{2}} \cdot \vec{E}{ }_{1}$ of the gates which it has passed through and

234 is smaller/equal to $\frac{\nabla|\vec{E}|^{2}}{E^{2}} \cdot \vec{E}_{2}$ of the gates where it is captured. In this way, insulin vesicles are separated in the 235 microfluidic channel, thus measuring the EKMr values for the insulin vesicles.

\section{RESULTS}

\section{Analysis of enriched insulin vesicle samples post fractionation}

The INS-1E cells used in these studies were capable of insulin secretion in response to increasing concentrations of glucose (Fig. S1). Isolated membrane fractions from differential and density gradient centrifugations

241 were analyzed through ELISA, western blotting (WB) and dynamic light scattering (DLS). ELISAs identified that

242 lower fractions of the density column (9-12) contained the highest insulin content (Fig. S2A), the same fractions

243 shown to be enriched in the insulin vesicle marker, synaptotagmin V, by WB (Fig. S2B). DLS indicated that these 244 fractions contained particles of 150-200 nm radius, which corresponds to the known range of insulin vesicles radii ${ }^{46,47}$.

245 Although the WB revealed that the final enriched vesicle sample included some contaminants from unwanted 246 organelles, such as the ER (Fig. S2B), this will not confound the analysis of insulin vesicles in the iDEP device as the 247 fluorescent labeling was targeted only at insulin vesicles. 


\section{Introduction of DC-iDEP as a discovery and quantification tool for insulin vesicle subpopulations}

Enriched vesicle samples from INS-1E cells were subjected to analysis using DC-iDEP after labeling with a high voltage (2100V, empirically determined, Fig. 3) such that all particles were prevented from entering the first gate, because DEP forces exceed EK forces. The voltage was then lowered incrementally (-300V for each step) allowing various subpopulations to enter the separation zone and be sorted along the device according to their specific EKMr values (Fig. 3). A bolus forms at a gate, corresponding to an EKMr value that is a result of a balance between DEP and EK forces on each particle and reflects a complex set of biological, chemical, and biophysical properties of the vesicles ${ }^{15,48-50}$. The fluorescence intensity was captured for each gate over a full range of voltages (1800V to $\left.600 \mathrm{~V}\right)$, such that the largest EKMr values are probed with the higher applied voltage (Fig. 4).

\section{Biophysical subpopulations of vesicles from untreated $\beta$-cells}

The distribution of fluorescently labeled n-insulin vesicles captured at each gate formed a characteristic arc

(Fig. 1C), indicating a well-operating and consistent system. Higher voltages provide a broader dynamic range of EKMr values for capturing a wider range of particles, while lower voltages provide detailed distribution of particles based on their associated EKMr values. At an applied voltage of $1800 \mathrm{~V}$, particles were sensed at EKMr values below $1.5 \times 10^{10} \mathrm{~V} / \mathrm{m}^{2}$ in patterns of overlapping subpopulations (Fig. 4A, blue circles). These overlapping features begin to spread out with an applied voltage of $1500 \mathrm{~V}$. At incrementally lower settings of applied voltages $(1200,900$, and 600V), distinctive patterns become identifiable (Fig. 4B-E). Notable and discernible features of bioparticle distribution are apparent around $1.2 \times 10^{10} \mathrm{~V} / \mathrm{m}^{2}$ and $1.8 \times 10^{10} \mathrm{~V} / \mathrm{m}^{2}$ at an applied voltage of $1200 \mathrm{~V}$ (Fig. 4C). Lowering the voltage to $900 \mathrm{~V}$, and redistribution of bioparticles based on adapted properties of the channel, reveals a similar but attenuated

271 feature of the distribution around $1.2 \times 10^{10} \mathrm{~V} / \mathrm{m}^{2}$ (Fig. 4D), whereas particles with EKMr values greater than $1.5 \times 10^{10}$

$272 \mathrm{~V} / \mathrm{m}^{2}$ leave the channel at this voltage. At this voltage, redistribution of particles, previously retained in overlapping 273 patterns at $1200 \mathrm{~V}$, forms a distinct peak around 5-6 $\times 10^{9} \mathrm{~V} / \mathrm{m}^{2}$ (Fig. 4D). Further lowering the voltage to $600 \mathrm{~V}$ shows 274 similar patterns of particle distribution around $5-6 \times 10^{9} \mathrm{~V} / \mathrm{m}^{2}$, as well as distinctive patterns around $3-4 \times 10^{9} \mathrm{~V} / \mathrm{m}^{2}(\mathrm{Fig}$. $2754 \mathrm{E})$, while leaving out populations with EKMr values higher than $1.0 \times 10^{10} \mathrm{~V} / \mathrm{m}^{2}$. 

same method used for the untreated cells (Fig. 4, yellow squares). Consistent with the vesicles from untreated cells, patterns of primarily overlapping subpopulations were detectable at a voltage of $1800 \mathrm{~V}$. Distribution of particles were observed at values up to $2.3 \times 10^{10} \mathrm{~V} / \mathrm{m}^{2}$ (compared to a maximum value of $1.5 \times 10^{10} \mathrm{~V} / \mathrm{m}^{2}$ for the untreated populations) (Fig. 4A), which suggests that these vesicles have a broader range of properties than the population from the untreated cells. The first evidence of a distinct distribution feature was captured around $7-8 \times 10^{9} \mathrm{~V} / \mathrm{m}^{2}$ when the voltage was lowered to $1500 \mathrm{~V}$ (Fig. 4B). Decreasing the voltage to $1200 \mathrm{~V}$ and subsequent particle redistribution in the channel revealed a distribution pattern with discernible features around $7-8 \times 10^{9} \mathrm{~V} / \mathrm{m}^{2}$, like those observed at $1500 \mathrm{~V}$, as well as a distinct peak around $1.1 \times 10^{10} \mathrm{~V} / \mathrm{m}^{2}$ (Fig. 4C). Further lowering the voltage to $900 \mathrm{~V}$ resulted in a unique distribution pattern with discernible features around $1.1 \times 10^{10} \mathrm{~V} / \mathrm{m}^{2}$ (Fig. 4D), similar to $1200 \mathrm{~V}$, and $8 \times 10^{9} \mathrm{~V} / \mathrm{m}^{2}$ (Fig. 4D), previously observed at $1500 \mathrm{~V}$ and $1200 \mathrm{~V}$ (Fig. 4B-C). Another feature of this distribution pattern was a peak around $4 \times 10^{9} \mathrm{~V} / \mathrm{m}^{2}$ (Fig. 4D). Ultimately, decreasing the voltage to $600 \mathrm{~V}$ revealed a distribution pattern with features around $4 \times 10^{9} \mathrm{~V} / \mathrm{m}^{2}$ and $8 \times 10^{9} \mathrm{~V} / \mathrm{m}^{2}$ (Fig. 4E), like those observed at higher voltages, leaving out patterns that were observed at EKMr values higher than $1.0 \times 10^{10} \mathrm{~V} / \mathrm{m}^{2}$. This distinct distribution pattern, although consistent with distribution patterns at higher voltages, featured stretched-out peaks, consistent with smaller and more refined range of EKMr values assigned throughout the channel.

\section{Behavior comparison of insulin vesicles and glucose stimulated insulin vesicles}

Visual inspection of the collected data (Fig. 4) indicated clear differences in the patterns of the insulin vesicle

297 distributions between the vesicle samples from treated and untreated cells. The n-insulin vesicles were discernible at

$298 \sim 1.2 \times 10^{10} \mathrm{~V} / \mathrm{m}^{2}, \sim 5-6 \times 10^{9} \mathrm{~V} / \mathrm{m}^{2}$, and $\sim 3-4 \times 10^{9} \mathrm{~V} / \mathrm{m}^{2}$ (weak at $\sim 1.8 \times 10^{10} \mathrm{~V} / \mathrm{m}^{2}$ ), whereas the g-insulin vesicles were

299 observed at $\sim 1.1 \times 10^{10} \mathrm{~V} / \mathrm{m}^{2}, \sim 8 \times 10^{9} \mathrm{~V} / \mathrm{m}^{2}$, and $\sim 4 \times 10^{9} \mathrm{~V} / \mathrm{m}^{2}$ (weak at $\sim 2.1 \times 10^{10} \mathrm{~V} / \mathrm{m}^{2}$ ). Beyond visual inspection,

300 two statistical methods were used to quantify and more deeply explore both the global and local differences: 1) the 301 likelihood of the two multi-component samples being different in composition, and 2) direct comparisons of paired 302 data points between the n-insulin vesicles and g-insulin vesicles data sets. To aid in deciding whether two complex 
whole data set for the largest differentiation without necessarily identifying the specifics. The criterion is based on the following equation (where the resulting values of $T$ can be correlated to a probability of the null result):

where $F_{N}\left(x_{i}\right)$ and $G_{M}\left(x_{i}\right)$ are the empirical distributions of the n- and g-insulin vesicles samples, the variables $x_{i}$ and $y_{i}$ are the observed values in each sample, and $N$ and $M$ represent the number of data points used to compare n- and g-insulin vesicles samples. An individual assessment was made for each applied voltage and indicated significant differences, consistent with the visual inspection. The calculated $T$ values were $4.8 \times 10^{3}$ at $600 \mathrm{~V}, 4.7 \times 10^{3}$ at $900 \mathrm{~V}$, $1.3 \times 10^{4}$ at $1200 \mathrm{~V}, 4.7 \times 10^{3}$ at $1500 \mathrm{~V}$ and $1.0 \times 10^{4}$ at $1800 \mathrm{~V}$. These values indicate that at all voltages, there is a larger than $99.9 \%$ confidence level that the null hypothesis that the two samples come from the same distribution can be rejected. This confidence level is even closer to $100 \%$ at $1200 \mathrm{~V}$ and $1800 \mathrm{~V}$ than other voltages. Further, a student's $\mathrm{t}$-test ${ }^{52}$ for paired data points of the intensity at each gate confirmed a statistical difference between $\mathrm{n}$ - and g-vesicles from the EKMr ranges of $3.25-7.45 \mathrm{~V} / \mathrm{m}^{2}$ at $1200 \mathrm{~V}$ (Fig. 4C), $0-1.40 \times 10^{9} \mathrm{~V} / \mathrm{m}^{2}$ at $1500 \mathrm{~V}$ and $1200 \mathrm{~V}$ (Fig. 4B-C), 5.60-7.15 $\times 10^{9} \mathrm{~V} / \mathrm{m}^{2}$ at $900 \mathrm{~V}$ (Fig. 4D), and $11.25-14.35 \times 10^{9} \mathrm{~V} / \mathrm{m}^{2}$ at $1800 \mathrm{~V}$ (Fig. 4A).

\section{DISCUSSION}

Using the DC-iDEP separation method, we identified distinct distribution patterns of insulin vesicles with varying biophysical properties which we interpret as indicative of vesicle subpopulations. Furthermore, we observed different distribution patterns for insulin vesicles isolated from glucose-stimulated versus unstimulated cells, which suggests glucose stimulation alters the insulin vesicle subpopulations. This is consistent with findings from single cell analysis using cryo-electron tomography and soft X-ray tomography, which revealed heterogeneities in the 326 composition of insulin vesicles ${ }^{53}$ and their molecular densities ${ }^{44}$ depending on the drug treatment and location of 327 insulin vesicles within the cell. Other studies have also observed enrichment of certain subpopulations of vesicles in 328 response to glucose stimulation ${ }^{54}$. Distinct subpopulations that were observed in both $\mathrm{n}$ - and g-insulin vesicles across 329 a wide range of EKMr values indicate that both $\mathrm{n}$ - and g-insulin vesicles are made up of complex and heterogenous subpopulations and are statistically different in their overall distributions pattern as well as in several specific EKMr 331 values. Our approach addresses an important need to identify and separate distinct subpopulations of insulin vesicles 332 which can allow for investigating their apparent heterogeneity and further characterizing them. 
The pattern of EKMr values is influenced by individual insulin vesicles. The distribution of this pattern reflects differences in physical properties of the vesicles in response to exposure of the cells to glucose. The specific changes to the individual insulin vesicles which result in varied EKMr measurements can be associated with any alteration of the biochemical makeup of the bioparticle. There is an ongoing evolution of the theoretical underpinnings of electric field gradient techniques, where past physical descriptors were limited to conductivity and permittivity of the particle. ${ }^{20}$ It is becoming better understood that the overall structure and subtle details of the bioparticle, including the particle-solvent cross polarizations, will influence these forces ${ }^{20-22,55,56}$. This new view of the forces imparted on the insulin vesicles aligns with a rather simple proposition that the makeup of the particle has changed: something has been added, subtracted, or altered which changed the force on the particle in a measurable and quantifiable way. This is consistent with findings that glucose can affect the maturation of existing vesicles, increasing their molecular density or the concentration of biomolecules within the vesicle lumen ${ }^{44}$. Additionally, the subpopulations we observe could have been altered by a change in surface protein expression or enrichment of unsaturated lipids ${ }^{57}$. This is also consistent with findings that glucose enhances vesicle-mitochondria association which is hypothesized to contribute to insulin vesicle maturation ${ }^{44}$. Identical particles always have the same EKMr in the same manner that identical proteins always have the same molecular weight ${ }^{58}$. $1.5 \times 10^{10} \mathrm{~V} / \mathrm{m}^{2}$ for the $\mathrm{n}$-vesicles, despite having identifiable populations with EKMr values higher than $1.5 \times 10^{10} \mathrm{~V} / \mathrm{m}^{2}$ at lower voltages. Another feature that is quite apparent is a lack of distinct 'peaks' or identifiable patterns appearing at consistent EKMr values within the data sets from differing applied voltage values. While the applied voltage does not affect the properties of bioparticles, it defines the forces that oppose bioparticle movements across the channel at different gates. Accordingly, in an overlapping pattern of subpopulations, those with higher EKMr values overcome the weaker opposing forces at a given gate once the voltage is lowered. ${ }^{16,59}$ Hence, the signal which was previously

357 Considering the high resolution of the technique, homogeneous subpopulations of vesicles undoubtably consist of a 358 very narrow range of EKMr values. Each data point shown in Figure 4 represents a homogenous subpopulation captured at the gate. While this feature is unsatisfying to classic separation scientists, it still allows for quantitative 
channels including the gate size and periodicity, as well as scan even more refined ranges of voltages to induce varying spectra of EKMr values along the device ${ }^{14,15}$. Additionally, developing the capability to port the collected individual boluses will enable downstream analyses such as mass spectrometry or electron microscopy. complex organelles subpopulations and study their distinctive distribution patterns under different treatment conditions with the goal of organelle subpopulation discovery and quantification. Further, the putatively subtle differences in subpopulations between stimulated and unstimulated $\beta$-cells are quantified more extensively than previously possible. Finally, this method provides a mechanism for the isolation and concentration of fractions which show the largest difference between the two population patterns for further bioanalysis (imaging, proteomics, lipidomics, etc.) that otherwise would not be possible given the low-abundance components of these subpopulations.

371 This approach can be broadly applied to any cell type and organelle beyond the scope of our model system of insulin 372 vesicles and $\beta$-cells.

\section{Author Contribution}

375 KLW, SEF, MAH and MB conceived the project. MB performed treatment on cells, harvested the cells, and performed 376 insulin vesicle enrichment followed by QC with ELISA, WB, and DLS. ZW performed fluorescent microscopy. YL 377 and MAH designed the iDEP experiments. YL performed all iDEP experiments, data reduction and presentation, and 378 conceived the statistical analysis. MB and YL performed statistical analysis on the iDEP data. MAH conceived the 379 Figures and MB created the Figures with input from all authors. All authors participated in data interpretation. All authors helped write and edit the manuscript, with YL and MB as leads.

\section{Competing Interest Statement}

383 MAH declares a conflict of interest with CBio where he serves as the CSO and with Hayes Diagnostics, Inc. where he serves as $\mathrm{COB}, \mathrm{CEO} \& \mathrm{CSO}$. All other authors have no conflict of interest to declare.

\section{Acknowledgements}

387 The authors would like to thank Raymond Stevens and the Bridge Institute for supporting our project and members of the Pancreatic $\beta$ Cell Consortium for their inspiring discussions and feedback. We additionally thank Chris Hanson 
389 for assisting with the cell culture, Yekaterina Kadyshevskaya for helping with illustrations, Claire Cato for feedback

390 on the manuscript, Brett Barbaro for assisting with analysis of the existing proteomics data, and USC NanoBiophysics

391 Core Facility for facilitating the DLS experiments.

392

\section{$393 \quad$ Funding}

394 This work was supported by the Bridge Institute at the USC Michelson Center for Convergent Bioscience (KLW) and 395 National Institutes of Health grant 5R03AI133397-02 (MAH). 

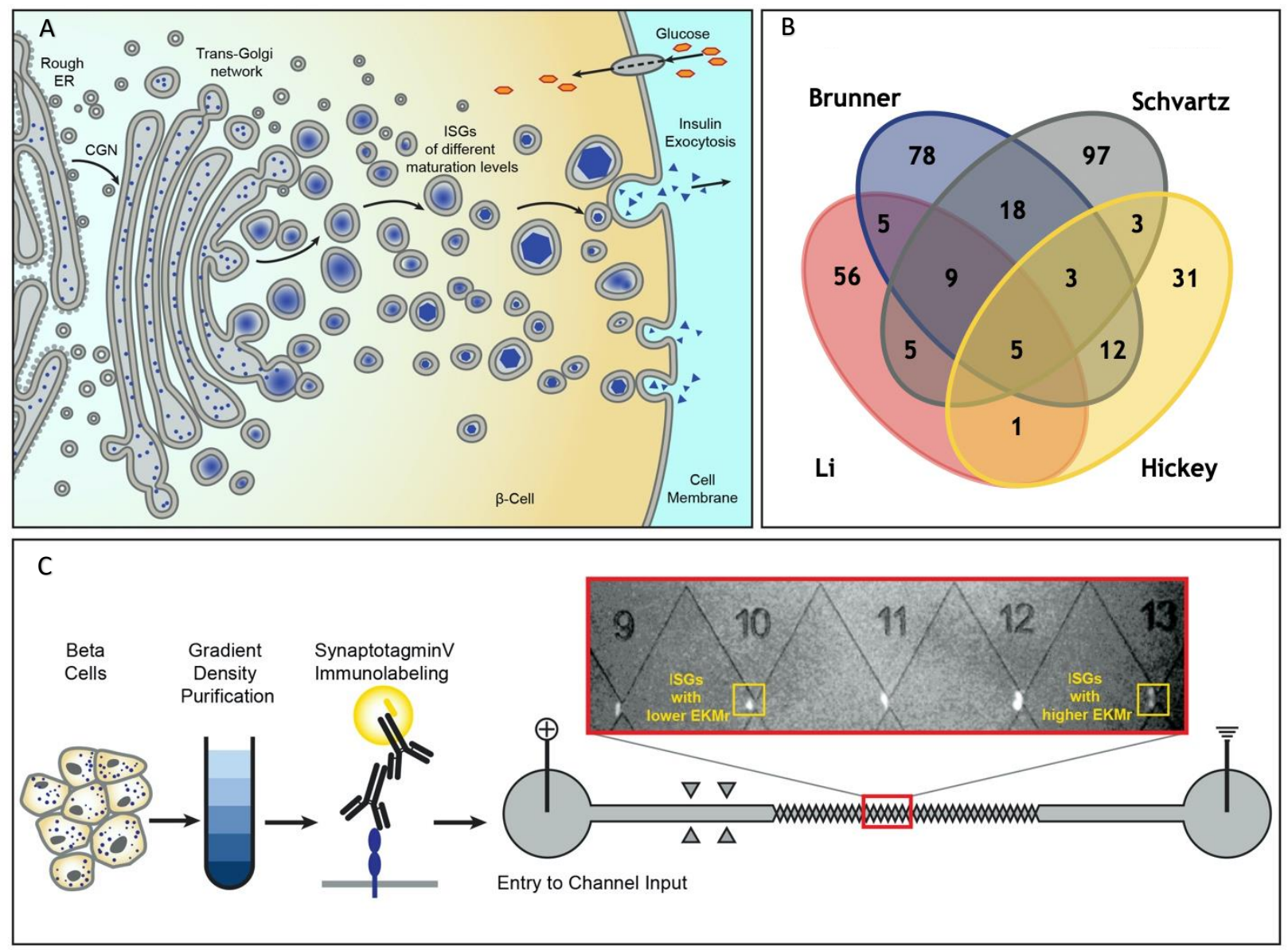

Figure 1. Schematic diagram for the formation of heterogenous insulin vesicles in pancreatic $\beta$-cells, graphical summation of disparate protein identifications, and processing of insulin vesicles including DC-iDEP device. A, insulin vesicle formation and maturation in a pancreatic $\beta$ cell. Newly synthesized insulin is packed inside secretory vesicles which mature to store crystalline insulin in vesicles until secretion is stimulated through different signaling pathways. B, four published insulin vesicle proteomics studies ${ }^{39-42}$ aimed to identify the proteome of the heterogenous populations of secretory vesicles in $\beta$-cells with only 5 proteins identified consistently. $\mathrm{C}$, separation of insulin vesicles using a DC-iDEP device. Differential and density gradient centrifugation were used to enrich each sample for insulin vesicle populations. Samples were then immunolabeled and introduced into DC-iDEP device for high resolution separation. Fluorescently labeled particles trapped near various gates in the channel are biophysically different subpopulations with varied EKMr (see text) values. The gates were constricted by increasing sizes of paired triangles, 

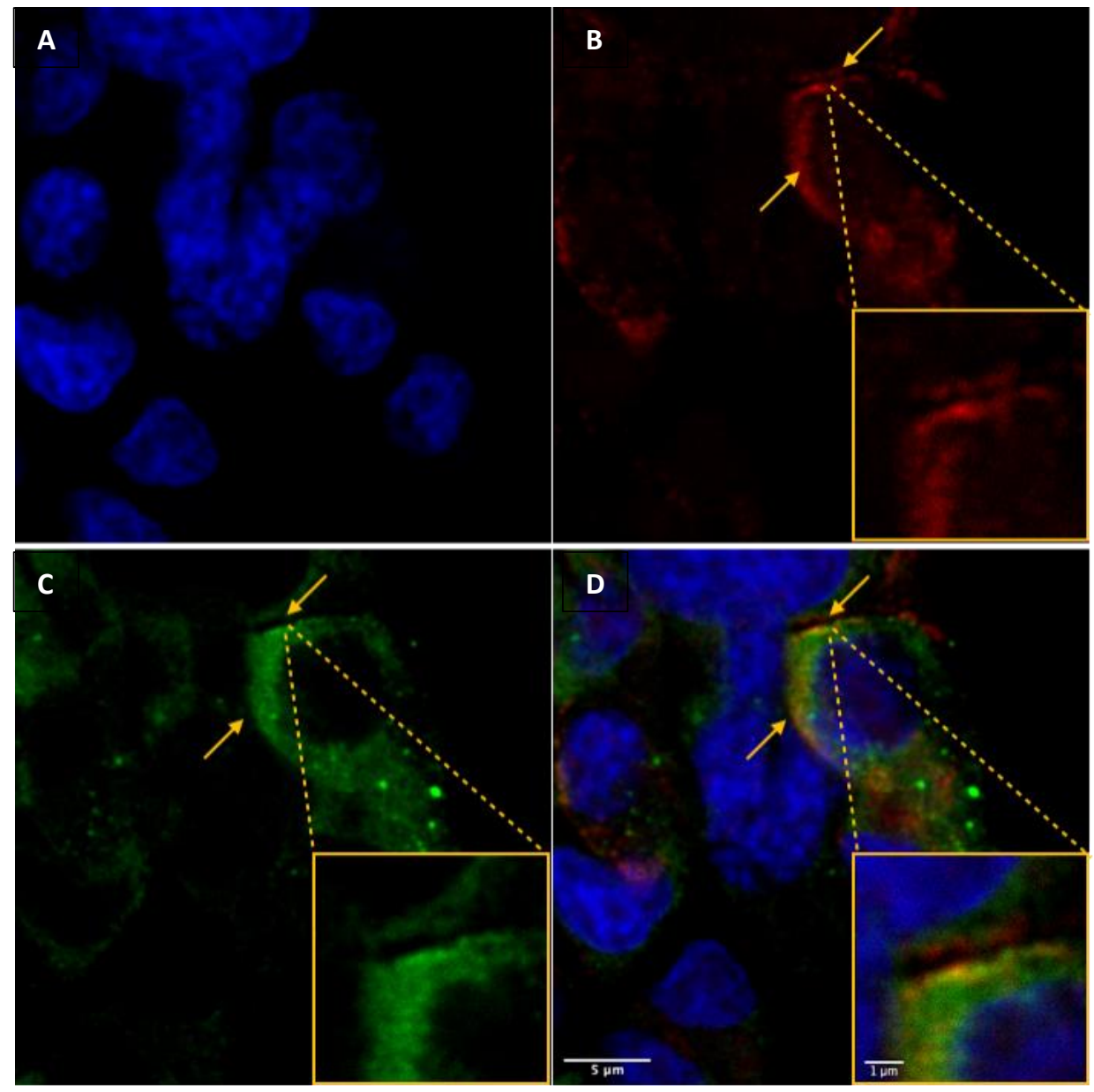

Figure 2. Colocalization of insulin vesicle marker used in this study (synaptotagmin V) with insulin. A, nuclei of INS$1 \mathrm{E} \beta$-cells stained with NucBlue ${ }^{\mathrm{TM}}$. B, synaptotagmin $\mathrm{V}$ labeled with mouse anti-synaptotagmin $\mathrm{V}$ and goat antimouse IgG $(\mathrm{H}+\mathrm{L})$, Alexa 647. C, hormone insulin labeled with rabbit anti-insulin and goat anti-rabbit $\operatorname{IgG}(\mathrm{H}+\mathrm{L})$, Alexa 488. D, localization of synaptotagmin $\mathrm{V}$ to insulin vesicles as apparent from the merged intensities of panels B and C. Microscopy was performed with a Leica microscope using a 63x/1.4NA oil immersion objective on cells mounted in ProLong ${ }^{\mathrm{TM}}$ Glass Antifade Mountant. 

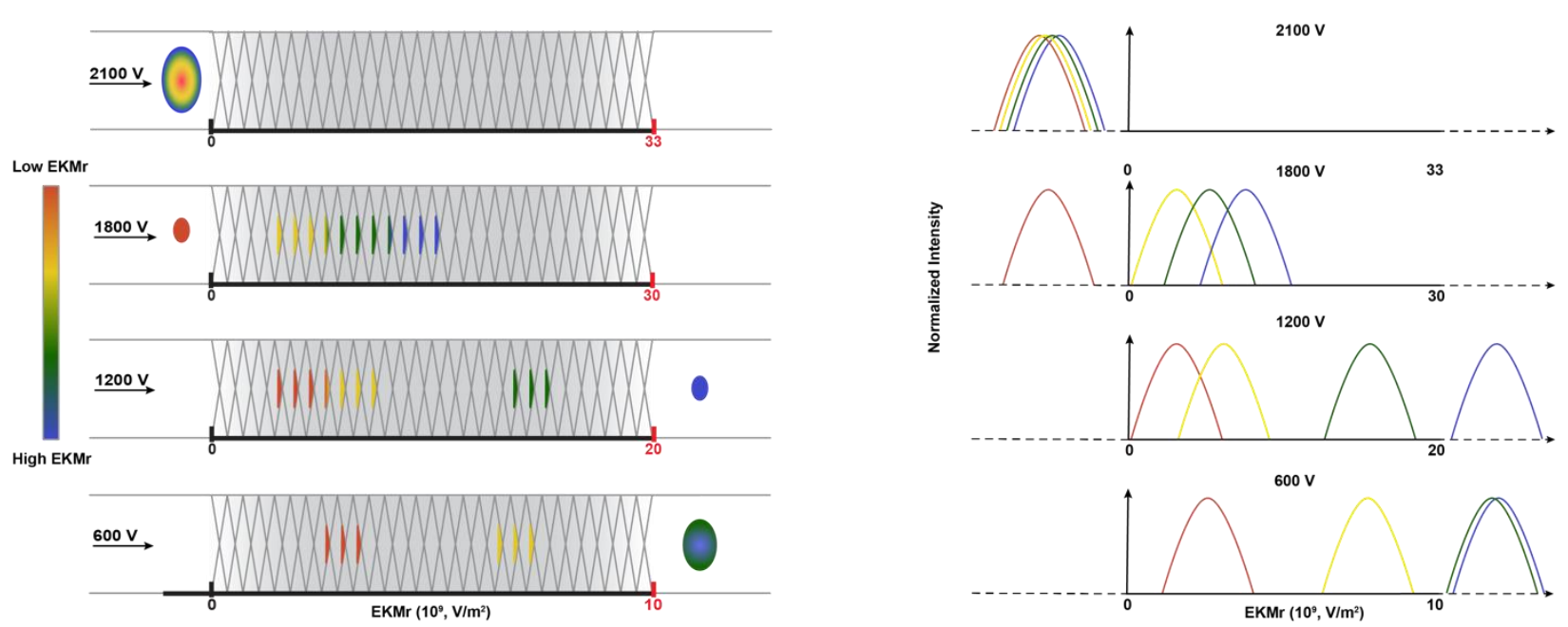

421 Figure 3. Schematic diagram of DC-iDEP system operated in a discovery or scanning mode. A, as a function of the design of the sawtooth channel with different gate sizes along the channel, the applied voltage defines DEP and EK forces at each gate, and capture occurs when the EK force of the particle is equal to or smaller than the DEP force. At high voltages, only particles with high EKMr values can enter the channel; the highest applied voltage of $2100 \mathrm{~V}$ prevents all particles of the sample from entering the inlet of the device due to the induced dielectrophoretic forces (no fluorescent signal detected anywhere along the channel). Sequentially lower voltages allow the various subpopulations to enter and be separated throughout the channel. When a subpopulation's EKMr value surpasses the channel's DEP force limit, it travels freely and leaves the channel at the outlet. B, fluorescent intensities of the captured particles are recorded along the channel at each voltage. Tracking these intensities allows the discovery and quantification of unknown subpopulations according to their biophysical properties. 

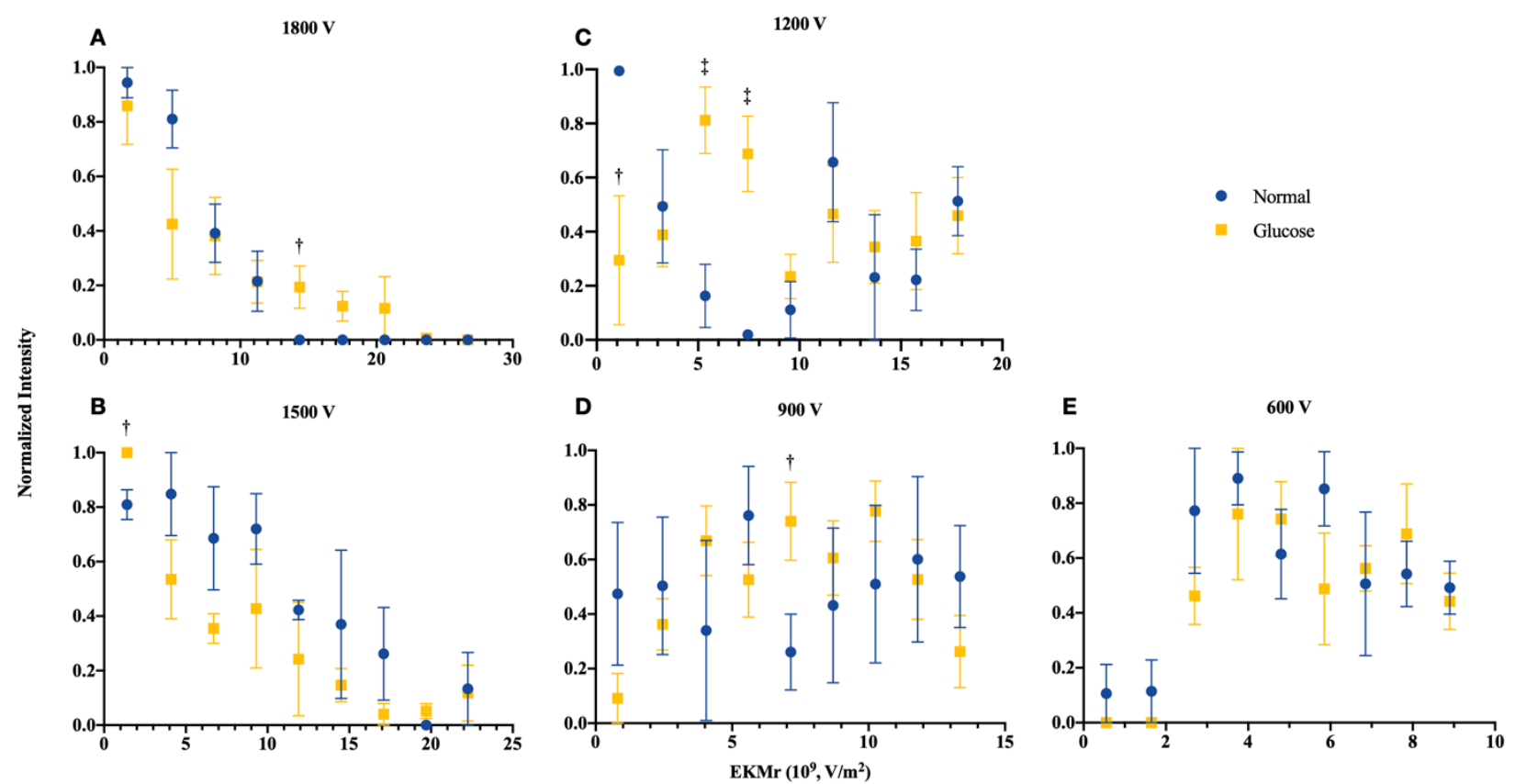

Figure 4. Comparison of distributions for the biophysical properties as reflected in EKMr values of n-insulin vesicles (blue circles) and g-insulin vesicles (yellow squares) with varied voltages applied. Fluorescent intensities of captured $\mathrm{n}$ - and g-insulin vesicles with different EKMr values were recorded at each gate. Each data point reflects fluorescent intensities recorded at 3 subsequent gates with the same EKMr values, averaged out over biologically replicated experiments and normalized over all signals recorded at a given voltage. Full profile of the sample's biophysical distribution was recorded at $1800 \mathrm{~V}$. Insulin vesicle subpopulations were separated at subsequent applied voltages of 1500, 1200, 900, and 600V. Values are mean \pm SEM ( $=3$ for $n$-vesicles or 4 for g-vesicles for biologically independent experiments). The two sample Cramer-von Mises Criterion shows that there is a larger than $99.9 \%$ probability that the $n$-insulin vesicles and g-insulin vesicles distribution differs at all voltages. Data are unity-based normalized and analyzed using student t-test to determine significant difference between data points of the two conditions. The t-test shows the $\mathrm{n}$ - and g-vesicles from the 3.25-7.45 V/m² EKMr range (C) differ significantly at $95 \%$ confidence level $(\$ \mathrm{P}<0.05)$. This test also shows that at the $90 \%$ confidence level $(\dagger \mathrm{P}<0.10)$, the n- and g-vesicle data points differ at the EKMr ranges of $0-1.40 \times 10^{9} \mathrm{~V} / \mathrm{m}^{2}(\mathrm{~B}-\mathrm{C}), 5.60-7.15 \times 10^{9} \mathrm{~V} / \mathrm{m}^{2}$ (D), and $11.25-14.35 \times 10^{9} \mathrm{~V} / \mathrm{m}^{2}(\mathrm{~A})$. 


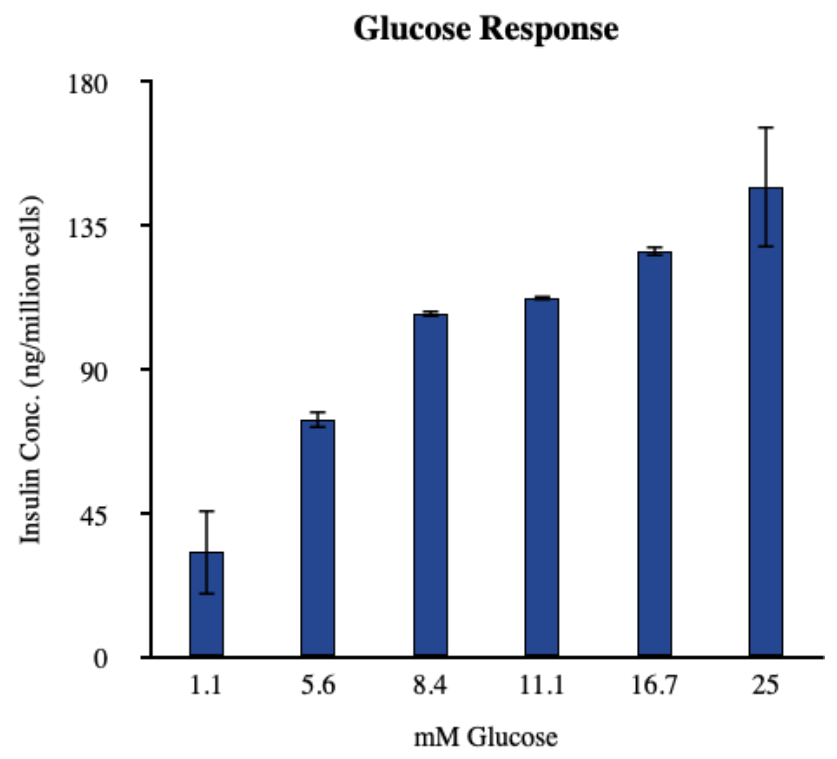

Figure S1. Glucose sensitivity of INS-1E $\beta$-cells was tested by stimulation at increasing concentrations of glucose and measurement of insulin secretion by ELISA. 


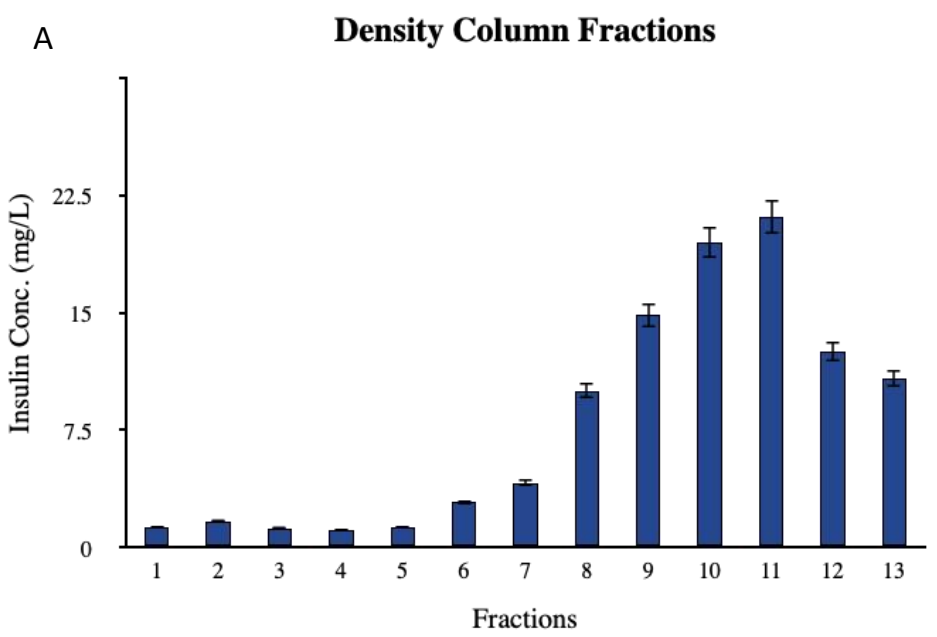

B

WB

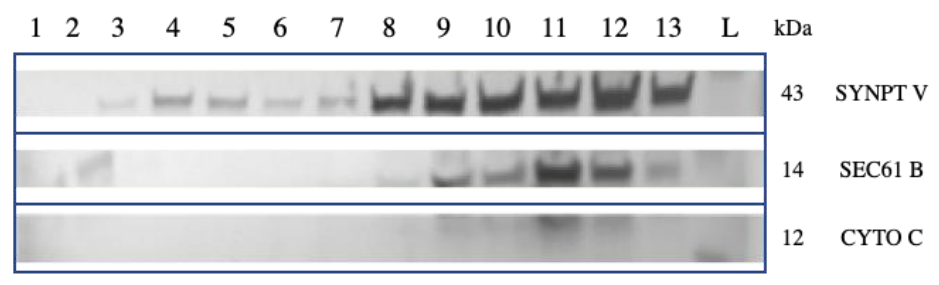

C

Dynamic Light Scattering

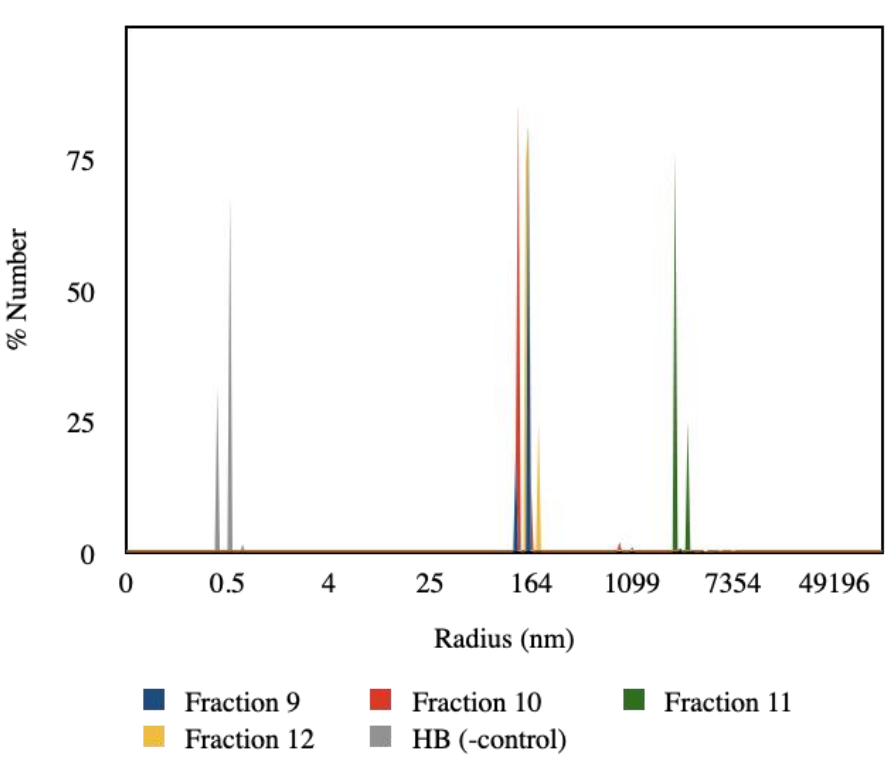

Figure S2. A, fractions of the density column were screened for insulin content in the ELISA assay. B, western blotting of the density column fractions revealed high concentrations of insulin vesicle marker synaptotagmin $\mathrm{V}$ in fractions with high insulin content as well as presence of ER and mitochondria contaminants as indicated by organelle markers SEC61 and Cytochrome C, respectively, in the same fractions. C, DLS was performed on fractions of interest 457 to validate presence of particles of $150-200 \mathrm{~nm}$ in radius, corresponding to radii of insulin vesicles. 
$501 \quad 17$ Liu, Y. et al. Identification of neural stem and progenitor cell subpopulations using DC insulator-based dielectrophoresis. Analyst 144, 4066-4072, doi:10.1039/C9AN00456D (2019).

50418 Hilton, S. H., Crowther, C. V., McLaren, A., Smithers, J. P. \& Hayes, M. A. Biophysical

508 differentiation of susceptibility and chemical differences in Staphylococcus aureus. Analyst 145, 2904-2914 (2020).

19 Chen, K. P., Pacheco, J. R., Hayes, M. A. \& Staton, S. J. R. Insulator-based dielectrophoretic separation of small particles in a sawtooth channel. Electrophoresis 30, 1441-1448, doi:10.1002/elps.200800833 (2009). electrical impedance studies of biomacromolecules. Electrophoresis 40, 2575-2583 (2019).

21 Matyushov, D. V. Electrostatic solvation and mobility in uniform and non-uniform electric fields: From simple ions to proteins. Biomicrofluidics 13, 064106 (2019).

22 Hayes, M. A. Dielectrophoresis of proteins: experimental data and evolving theory. Analytical and bioanalytical chemistry, 1-11 (2020).

23 Kim, D., Sonker, M. \& Ros, A. Dielectrophoresis: From Molecular to Micrometer-Scale Analytes. Anal Chem 91, 277-295, doi:10.1021/acs.analchem.8b05454 (2019). Lapizco-Encinas, B. H. \& Rito-Palomares, M. Dielectrophoresis for the manipulation of nanobioparticles. Electrophoresis 28, 4521-4538 (2010). pathway marker, in INS-1 pancreatic beta cells. J Biol Chem 278, 31486-31494, doi:10.1074/jbc.M305690200 (2003). occurs within immature beta-granules. Journal of Biological Chemistry 269, 20838-20844 (1994). in $\beta$-cells. Mol Metab 6, 958-973, doi:10.1016/j.molmet.2017.04.010 (2017). Orci, L. The insulin factory: a tour of the plant surroundings and a visit to the assembly line. Diabetologia 28, 528-546, doi:10.1007/BF00281987 (1985). Orci, L. et al. Conversion of proinsulin to insulin occurs coordinately with acidification of maturing secretory vesicles. The Journal of cell biology 103, 2273-2281, doi:10.1083/jcb.103.6.2273 (1986).

30 Dean, P. M. Ultrastructural morphometry of the pancreatic $\beta$-cell. Diabetologia 9, 115119, doi:10.1007/BF01230690 (1973).

31 Hou, J. C., Min, L. \& Pessin, J. E. Insulin granule biogenesis, trafficking and exocytosis. Vitam Horm 80, 473-506, doi:10.1016/S0083-6729(08)00616-X (2009).

32 Michael, D. J., Ritzel, R. A., Haataja, L. \& Chow, R. H. Pancreatic beta-cells secrete insulin in fast- and slow-release forms. Diabetes 55, 600-607, doi:10.2337/diabetes.55.03.06.db05-1054 (2006). distinct calcium sources. Journal of Cell Science 118, 5873, doi:10.1242/jcs.02684 (2005). pancreatic islets. Endocrinology 110, 1183-1188, doi:10.1210/endo-110-4-1183 (1982). 
54535 Gold, G., Gishizky, M. L. \& Grodsky, G. M. Evidence that glucose \&quot;marks\&quot;

36 Thurmond, D. C. in Mechanisms of Insulin Action: Medical Intelligence Unit 52-70 (Springer New York, 2007).

37 Hutton, J. C., Penn, E. J. \& Peshavaria, M. Isolation and characterisation of insulin secretory granules from a rat islet cell tumour. Diabetologia 23, 365-373, doi:10.1007/BF00253746 (1982).

39 Schvartz, D. et al. Improved characterization of the insulin secretory granule proteomes. Brune, D. \& Kim, S. Predicting Protein Diffusion Coefficients. Proceedings of the National Academy of Sciences of the United States of America 90, 3835-3839, J Proteomics 75, 4620-4631, doi:10.1016/j.jprot.2012.04.023 (2012). correlation profiling. Biophysics Reports 4, 329-338, doi:10.1007/s41048-018-0061-3 (2018). Hickey, A. J. et al. Proteins associated with immunopurified granules from a model pancreatic islet beta-cell system: proteomic snapshot of an endocrine secretory granule. J Proteome Res 8, 178-186, doi:10.1021/pr800675k (2009). Merglen, A. et al. Glucose sensitivity and metabolism-secretion coupling studied during two-year continuous culture in INS-1E insulinoma cells. Endocrinology 145, 667-678, doi:10.1210/en.2003-1099 (2004). Lin W, Tseng K, McDermott G, Alber F, Sali A, Larabell C, Stevens RC. Visualizing subcellular rearrangements in intact $\beta$-cells using soft $X$-ray tomography. Science Advances 6 (2020). Staton, S. J., Chen, K. P., Taylor, T. J., Pacheco, J. R. \& Hayes, M. A. Characterization of particle capture in a sawtooth patterned insulating electrokinetic microfluidic device. Electrophoresis 31, 3634-3641, doi:10.1002/elps.201000438 (2010).

Olofsson, C. S. et al. Fast insulin secretion reflects exocytosis of docked granules in mouse pancreatic B-cells. Pflügers Archiv 444, 43-51, doi:10.1007/s00424-002-0781-5 (2002). from rat islets of Langerhans. II. Ultrastructure of the beta granule. The Journal of cell biology 41, 162-166, doi:10.1083/jcb.41.1.162 (1969).

4 Hilton, S. H. \& Hayes, M. A. A mathematical model of dielectrophoretic data to connect measurements with cell properties. Anal Bioanal Chem 411, 2223-2237, doi:10.1007/s00216-019-01757-7 (2019).

49 Crowther, C. V., Hilton, S. H., Kemp, L. \& Hayes, M. A. Isolation and identification of Listeria monocytogenes utilizing DC insulator-based dielectrophoresis. Analytica Chimica Acta 1068, 41-51, doi:https://doi.org/10.1016/j.aca.2019.03.019 (2019). 
$58850 \quad$ Liu, Y. M. \& Hayes, M. A. Differential Biophysical Behaviors of Closely Related Strains of

$589 \quad$ Salmonella. Front Microbiol 11, doi:ARTN 302

590 10.3389/fmicb.2020.00302 (2020).

$59151 \quad$ Anderson, T. W. On the Distribution of the Two-Sample Cramer-von Mises Criterion.

$592 \quad$ Ann. Math. Statist. 33, 1148-1159, doi:10.1214/aoms/1177704477 (1962).

59352 de Winter, J. C. F. Using the Student's t-test with extremely small sample sizes Practical

$594 \quad$ Assessment, Research, and Evaluation 18 ((2013)).

$59553 \quad$ Zhang X, C. S., Singla J, White KL, Butler PC, Stevens RC, Jensen GJ. Visualizing insulin

596 vesicle neighborhoods in beta-cells by cryo-electron tomography. Science Advances 6

597 (2020).

59854 Straub, S. G., Shanmugam, G. \& Sharp, G. W. G. Stimulation of Insulin Release by

$599 \quad$ Glucose Is Associated With an Increase in the Number of Docked Granules in the $\beta$-Cells

$600 \quad$ of Rat Pancreatic Islets. Diabetes 53, 3179, doi:10.2337/diabetes.53.12.3179 (2004).

60155 Hölzel, R. \& Pethig, R. Protein dielectrophoresis: Key dielectric parameters and evolving

602 theory. Electrophoresis (2020).

60356 Martin, D. R. \& Matyushov, D. V. Solvated dissipative electro-elastic network model of

$604 \quad$ hydrated proteins. The Journal of chemical physics 137, 165101 (2012).

60557 Moore, J. H. et al. Conductance-Based Biophysical Distinction and Microfluidic

$606 \quad$ Enrichment of Nanovesicles Derived from Pancreatic Tumor Cells of Varying

607

608 Invasiveness. Analytical Chemistry 91, 10424-10431, doi:10.1021/acs.analchem.8b05745

609 (2019).

610

611

612

Zhu, F., Nannenga, B. L. \& Hayes, M. A. Electrophoretic exclusion microscale sample preparation for cryo-EM structural determination of proteins. Biomicrofluidics 13,

613 054112 (2019).

614

59 Jones, P. V. \& Hayes, M. A. Development of the resolution theory for gradient insulatorbased dielectrophoresis. ELECTROPHORESIS 36, 1098-1106, 